DOI: $10.18027 / 2224-5057-2021-11-3 s 2-11$

Цитирование: Хохлова С. В, Давыдова И.Ю., Коломиец Л.А., Кузнецов В.В., Новикова Е.Г., Трякин А.А., Тюляндина А. и соавт. Практические рекомендации по лекарственному лечению злокачественных неэпителиальных опухолей яичников. Злокачественные опухоли: Практические рекомендации RUSSCO \#3s2, 2021 (том 11). 11

\title{
ПРАКТИЧЕСКИЕ РЕКОМЕНДАЦИИ ПО ЛЕКАРСТВЕННОМУ ЛЕЧЕНИЮ ЗЛОКАЧЕСТВЕННЫХ НЕЭПИТЕЛИАЛЬНЫХ ОПУХОЛЕЙ ЯИЧНИКОВ
}

Коллектив авторов: Хохлова С. В, Давыдова И.Ю., Коломиец Л.А., Кузнецов В.В., Новикова Е.Г., Трякин А.А., Тюляндина А.С., Урманчеева А.Ф.

Ключевые слова: герминогенные опухоли, дисгерминома, стромальные опухоли, тератома, недисгерминома

\section{1. КЛАССИФИКАЦИЯ И ОПРЕДЕЛЕНИЕ СТАДИИ}

Стадирование злокачественных неэпителиальных опухолей яичников (ЗНЭОЯ) проводится так же, как и эпителиальных, по результатам интраоперационной ревизии и послеоперационного гистологического исследования; используются классификации TNM (8-е издание, 2017 г.) и FIGO (2009 г.) (табл. 1). Морфологическая классификация (ВОЗ 2020 г.) представлена в табл. 2.

Таблица 1. Система стадирования рака яичников по TNM (8-е издание, 2017 гг.) и FIGO (2009 г.)

\begin{tabular}{|l|l|l|}
\hline TNM & FIGO & \\
\hline T1 & I & Опухоль ограничена яичниками \\
\hline T1a & IA & $\begin{array}{l}\text { Опухоль ограничена одним яичником, капсула не повреждена, нет опухолевых } \\
\text { жазрастаний на поверхности яичника, нет злокачественных клеток в асцитической } \\
\text { жидкости или смывах из брюшной полости }\end{array}$ \\
\hline T1b & IB & $\begin{array}{l}\text { Опухоль ограничена двумя яичниками, их капсулы не повреждены, нет опухолевых } \\
\text { разрастаний на поверхности яичников, нет злокачественных клеток в асцитической } \\
\text { жидкости или смывах из брюшной полости }\end{array}$ \\
\hline T1с & IC & $\begin{array}{l}\text { Опухоль ограничена одним или двумя яичниками в сочетании с одним из следующих } \\
\text { факторов }\end{array}$ \\
\hline T1с1 & IC1 & Повреждение капсулы во время операции \\
\hline T1с2 & IC2 & Повреждение капсулы до операции или опухоль на поверхности яичника/маточной трубы \\
\hline T1с3 & IC3 & Злокачественные клетки в асцитической жидкости или смывахс брюшины \\
\hline T2 & II & Опухоль поражает один или два яичника с распространением на малый таз \\
\hline
\end{tabular}




\begin{tabular}{|c|c|c|}
\hline TNM & FIGO & \\
\hline $\mathrm{T} 2 \mathrm{a}$ & IIA & Врастание и/или метастазирование в матку и/или в одну или обе маточные трубы \\
\hline $\mathrm{T} 2 \mathrm{~b}$ & IIB & Распространение на другие ткани таза \\
\hline $\begin{array}{l}\text { T3 } \\
\text { и/или } \\
\text { N1 }\end{array}$ & III & $\begin{array}{l}\text { Опухоль поражает один или оба яичника с гистологически подтвержденными } \\
\text { внутрибрюшинными метастазами за пределами таза и/или метастазами в регионарных } \\
\text { лимфатических узлах (внутренних, наружных и общих подвздошных, запирательных, } \\
\text { крестцовых, поясничных или паховых лимфоузлах) }\end{array}$ \\
\hline N1 & $\begin{array}{l}\text { IIIA1 } \\
\text { IIIA1 (i) } \\
\text { IIIA (ii) }\end{array}$ & $\begin{array}{l}\text { Метастазы только в забрюшинных лимфатических узлах } \\
\text { Метастазы в лимфатических узлах размерами до } 10 \text { мм } \\
\text { Метастазы в лимфатических узлах размерами более } 10 \text { мм }\end{array}$ \\
\hline T3a & IIIA2 & $\begin{array}{l}\text { Микроскопические гистологически подтвержденные внутрибрюшинные метастазы } \\
\text { за пределами таза } \pm \text { метастазы в забрюшинных лимфатических узлах }\end{array}$ \\
\hline T3b & IIIB & $\begin{array}{l}\text { Макроскопические внутрибрюшинные метастазы за пределами таза до } 2 \text { см } \\
\text { включительно в наибольшем измерении } \pm \text { метастазы в забрюшинных лимфатических } \\
\text { узлах }\end{array}$ \\
\hline T3c & IIIC & $\begin{array}{l}\text { Внутрибрюшинные метастазы за пределами таза более } 2 \text { см в наибольшем измерении } \\
\text { метастазы в забрюшинных лимфатических узлах (включая распространение опухоли } \\
\text { на капсулу печени и селезенки без поражения паренхимы органов) }\end{array}$ \\
\hline M1 & $\begin{array}{l}\text { IV } \\
\text { IVA } \\
\text { IVB }\end{array}$ & $\begin{array}{l}\text { Отдаленные метастазы (исключая внутрибрюшинные метастазы) } \\
\text { Плевральный выпот со злокачественными клетками } \\
\text { Метастазы в паренхиматозных органах и других органах вне брюшной полости (в том } \\
\text { числе паховых лимфатических узлах и лимфатических узлах за пределами брюшной } \\
\text { полости) }\end{array}$ \\
\hline
\end{tabular}

Таблица 2. Морфологическая классификация неэпителиальных опухолей яичников (ВОЗ 2020 г., 5 издание)

\section{ОПУХОЛИ СТРОМЫ И ПОЛОВОГО ТЯЖА}

\section{Стромальные опухоли}

Фиброма

8810/1-клеточная фиброма

8600/0-текома

8601/0 - текома лютеинизирующая

8602/0 - склерозирующая стромальная опухоль

8590/0 - мелкокистозная стромальная опухоль

8590/0 - перстневидноклеточная стромальная опухоль

8650/0-опухоль из клеток Лейдига

8670/0 - стероидноклеточная опухоль

8670/3 - стероидноклеточная опухоль, злокачественная

8810/3 - фибросаркома

\section{Опухоли полового тяжа}

8620/3 - гранулезоклеточная опухоль взрослого типа

8622/1 - гранулезоклеточная опухоль ювенильного типа

8640/1- опухоль из клеток Сертоли

8623/1-опухоль полового тяжа с кольцевидными трубочками 


\section{Смешанные опухоли стромы и полового тяжа}

8631/1-опухоль из клеток Сертоли-Лейдига

8631/0 - опухоль из клеток Сертоли-Лейдига высокодифференцированная

8631/1-опухоль из клеток Сертоли-Лейдига умереннодифференцированная

8631/3 - опухоль из клеток Сертоли-Лейдига низкодифференцированная

8633/1- опухоль из клеток Сертоли-Лейдига ретиформная

8590/1- опухоль полового тяжа

8632/1-гинандробластома

ГЕРМИНОГЕННЫЕ ОПУХОЛИ

9080/0-тератома доброкачественная зрелая

9080/3 - незрелая тератома

9060/3-дисгерминома

9071/3 - опухоль желточного мешка

9100/3 - хориокарцинома

9070/3 - эмбриональный рак

9085/3 - смешанная злокачественная герминогенная опухоль

Монодермальные тератомы и опухоли соматического типа, развившиеся в дермоидной кисте

9090/0 - струма яичника

9090/3 - струма яичника злокачественная

9091/1- струмальный карциноид

9084/3 - тератома со злокачественной трансформацией

9080/0-кистозная тератома

Герминогенные опухоли в сочетании с опухолями стромы и полового тяжа

90731/1-гонадобластома

8594/1-смешанная герминогенная опухоль и опухоль стромы и полового тяжа

\section{2. ДИАГНОСТИКА}

План обследования при подозрении на ЗНЭОЯ включает:

- сбор анамнеза, изучение клинических симптомов;

- физикальное, в том числе гинекологическое исследование (бимануальное ректовагинальное исследование с цитологическим исследованием мазков с шейки матки);

- общий биохимический и общеклинический анализы крови, анализ мочи;

- определение уровня опухолевых маркеров и гормонов:

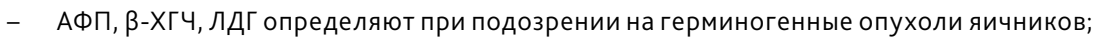

- ингибин В и мюллеровскую ингибирующую субстанцию/антимюллеров гормон следует определять при подозрении на опухоли стромы полового тяжа;

- тестостерон иэстрадиол может повышаться при опухолях из клеток Сертоли-Лейдига (вирилизация отмечается у $70-85$ \% больных) и других опухолях стромы полового тяжа;

- CA125 и НЕ-4 может незначительно повышаться при герминогенных опухолях, карциносаркомах яичников;

- СА 19-9 и РЭА могут незначительно увеличиваться при тератомах яичников. 
- R-логическое исследование органов грудной клетки; КТ органов грудной клетки - по показаниям;

- УзИ органов малого таза, брюшной полости и забрюшинного пространства/КТ органов брюшной полости и малого таза св/в контрастированием/МРТ органов брюшной полости и малого таза с в/в контрастированием;

- обследование ЖКТ (эндоскопическое или R-логическое);

- МРТ головного мозга (по показаниям); при высоком уровне $\beta$-Х ГЧ (>50000 мME/мл) или множественных метастазах в легких в связи с высоким риском поражения головного мозга;

- диагностическую гистероскопию, взятие аспирата из полости матки или выскабливание полости матки с гистологическим исследованием (при УЗИ-признаках гиперплазии эндометрия);

- у молодых пациенток с первичной аменореей необходимо генетическое исследование кариотипа с целью исключения дисгенезии гонад [синдрома Swire (XY-генотип) и дp.];

- остеосцинтиграфию (при подозрении на метастатическое поражение костей).

\section{3. ЛЕЧЕНИЕ}

\section{1. Опухоли стромы и полового тяжа (гранулезоклеточные опухоли составляют подавляющее большинство всех опухолей стромы полового тяжа)}

\subsection{1. Лечение ранних стадий опухолей стромы полового тяжа (ОСПт) (стадии IA, B, C)}

На первом этапе показано хирургическое лечение. Рекомендуемый хирургический доступ - лапаротомия. Лапароскопический доступ возможен в исключительных случаях на ранних стадиях заболевания, при опухолях небольших размеров при условии высокой квалификации хирурга и выполнения операции с учетом всехправил абластики. Больным менопаузального возраста рекомендована экстирпация матки с придатками.

Хирургическое стадирование включает резекцию большого сальника, биопсию подозрительных участков брюшины, взятие мазков с париетальной брюшины диафрагмы, латеральных каналов, малого маза, интраоперационное взятие смывов с брюшной полости для цитологического исследования.

Выполнение лимфодиссекции (тазовой и парааортальной) не является обязательным, однако следует помнить, что метастазы в лимфоузлах при гранулезоклеточных опухолях и других злокачественных опухолях стромы и полового тяжа могут встречаться.

У больных репродуктивного возраста при IA/C стадии ОСПТ, желающих сохранить фертильность, возможно выполнение односторонней аднексэктомии. Биопсию контралатерального яичника выполнять нецелесообразно, если он визуально не изменен.

С учетом высокой вероятности одностороннего поражения яичника при ОСПТ у больных репродуктивного возраста предпочтение отдается органосохраняющим объемам хирургического вмешательства. 
При ультразвуковых признаках гиперплазии эндометрия необходимо выполнять раздельное диагностическое выскабливание матки или аспирационную биопсию эндометрия для исключения рака эндометрия.

Больным I стадией ОСПТ с низким риском прогрессирования (целостность капсулы не нарушена, капсула опухоли интактна, по поверхности капсулы опухоли нет) рекомендовано динамическое наблюдение.

Больным I стадией ОСПТ с высоким риском прогрессирования (нарушена целостность капсулы опухоли, IC стадия, низкая дифференцировка опухоли) или промежуточным риском (гетерологичные элементы в ОСПТ) можно предложить два варианта ведения: наблюдение или адъювантнуюХТ.

В качестве адъювантной ХТ обычно применяется комбинация ВЕР (4 цикла) или паклитаксел +карбоплатин (4 курса), обладающих схожей эффективностью. У молодыхженщин (до 40 лет) отдается предпочтение режиму ВЕР.

Повторные стадирующие операции (удаление большого сальника, биопсия брюшины, взятие смывов из брюшной полости) рекомендованы, если данная процедура не была изначально выполнена.

Если первоначально выполнялась резекция опухолево измененного яичника у больных предположительно с I стадией заболевания, рекомендована повторная операция в объеме аднексэктомии (у молодых пациенток, желающих сохранить репродуктивную и гормональную функции) или пангистерэктомии (у больных в менопаузе или молодых пациенток, не желающих сохранять репродуктивную и гормональную функции) и выполнение этапов стадирования.

\subsection{2. Лечение распространенных стадий и рецидивов опухоли стромы и полового тяжа}

Женщинам с распространенными стадиями (стадии II-IV) рекомендовано на первом этапе хирургическое лечение в объеме лапаротомии, экстирпации матки с придатками, удаления большого сальника, взятие смывов и биопсии брюшины, тазовая, парааортальная лимфодиссекция по показаниям (подозрение на метастатическое изменение лимфоузлов).

Всем больным со II-IV стадиями ОСПТ рекомендовано проведение адъювантной XT (табл. 3). При наличии изолированной резидуальной опухоли в малом тазу возможно проведение лт.

Наиболее эффективными являются оптимальные циторедуктивные операции. При распространенных стадиях и рецидиве заболевания на I этапе выполняется хирургическое лечение с последующими 3-4 курсами ХТ по схеме ВЕР или 4 курсами паклитаксел +карбоплатин. Если ранее проводилась ХТ по схеме ВЕР, то возможны следующие комбинации лекарственного лечения: «паклитаксел +карбоплатин» или «циклофосфамид +доксорубицин +цисплатин» (табл. 3).

Определенной эффективностью обладает гормонотерапия (ингибиторы ароматазы (анастрозол, летрозол), мегестрола ацетат, провера, тамоксифен).

При локализованном рецидиве опухоли стромы и полового и тяжа возможно назначение лт. 
Иммунотерапия: пембролизумаб может применяться у больных с высокой MSI или c dMMR.

Гранулезоклеточные опухоли, несмотря на относительно благоприятный прогноз, рецидивируют сравнительно часто. Рецидивы могут возникать спустя годы, вплоть до 20 лет, что требует длительного наблюдения за больными, поскольку рецидив и прогрессирование становятся в дальнейшем причиной летальности этой категории больных.

\section{2. Герминогенные опухоли яичников}

В лечении злокачественных герминогенных опухолей яичников (ЗГОЯ) среди химиотерапевтов принято разделение всех герминогенных опухолей яичников на дисгерминомы и недисгерминомы. Повышенный уровень АФП и высокий уровень $\beta$-ХГЧ (> 200 мМЕ/л) при дисгерминоме свидетельствует о наличии элементов недисгерминомы (опухоли желточного мешка, хориокарциномы), что требует проведения терапии по принципам лечения недисгерминомы. Наличие повышенного уровня АФП или $\beta$-ХГЧ у пациенток со зрелой тератомой свидетельствует о наличии в опухоли злокачественного компонентанезрелой тератомы, опухоли желточного мешка, хориокарциномы и др. Такие больные должны быть направлены к химиотерапевту и в зависимости от гистологической формы, дифференцировки опухоли и стадии заболевания, будут определены показания для ХT.

\subsection{1. Первичное лечение зГОЯ}

Перед операцией желательно иметь информацию о маркерах герминогенных опухолей

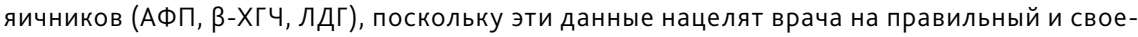
временный подход к лечению. Для этого всем пациенткам молодого и репродуктивного возраста с новообразованиями яичников необходимо исследовать уровень данных маркеров. Указать на возможный гистологический характер опухоли яичников, в частности герминогенный или ОСПТ с высокой долей вероятности способно квалифицированное ультразвуковое исследование. Хирургическое стадирование имеет большое значение для определения тактики последующего лечения. Особенно это касается дисгерминомы, при которой в случае I стадии можно избежать ХT. Хирургическое лечение включает срединную или нижнесрединную лапаротомию и вне зависимости от распространенности процесса - одностороннюю сальпингоовариэктомию у пациенток, желающих сохранить репродуктивную и гормональную функции. Пациенткам, не желающим сохранить репродуктивную и гормональную функции, а также находящимся в постменопаузе, рекомендуется экстирпация матки с придатками.

Так же как и при ОСПТ, лапароскопический доступ может применяться в исключительных случаях на ранних стадиях заболевания при опухолях небольших размеров при условии высокой квалификации хирурга и выполнения операции с учетом всех правил абластики. Повреждение капсулы опухоли, попадание содержимого опухоли в брюшную полость, на брюшину, касание опухоли портов, касание брюшины инструментами, контактирующими с опухолью, должно быть исключено. 
Срочное интраоперационное гистологическое исследование удаленного яичника является обязательным для определения адекватного хирургического объема. Резекция контралатерального яичника выполняется только при макроскопических признаках поражения. При дисгенезии гонад (кариотип XY устанавливается при обследовании генетиком) производят двустороннюю гонадэктомию. Необходимость выполнения оментэктомии при зГОЯ четко не определена вследствие редкости изолированного метастазирования в большой сальник. Тем не менее, выполнение оментэктомии по поперечной ободочной кишке рекомендовано с целью определения стадии заболевания и определения дальнейшей тактики лечения. Для определения стадии заболевания также обязателен забор смывов с париетальной брюшины диафрагмы, латеральных каналов, малого таза для дальнейшего цитологического исследования, производится биопсия подозрительных участков брюшины. Подвздошная и/или парааортальная лимфодиссекция выполняется только при наличии увеличенных лимфоузлов. Принцип хирургического лечения включает удаление всех видимых метастатических образований без резекции смежных органов. С учетом высокой чувствительности опухоли к ХТ объем циторедуктивного вмешательства не должен носить калечащего характера, а также не приводить к потенциальному росту частоты послеоперационных осложнений, которые могут задержать начало ХТ. Своевременное начало XT, соблюдение четких временных диапазонов и правильно установленные дозы химиопрепаратов являются залогом успеха в лечении герминогенных опухолей яичников.

При неадекватном стадировании повторная циторедукция с целью рестадирования рекомендуется только в том случае, если в дальнейшем может рассматриваться такая опция как наблюдение.

\subsubsection{2. Тактика лечения после хирургического лечения}

Дальнейшая лечебная тактика зависит от гистологического типа опухоли и стадии заболевания. В случае смешанного типа ЗГОЯ лечение планируется по наиболее неблагоприятному морфологическому варианту. После операции обязательно повторное определение уровней АФП и $\beta$-ХГЧ, если они были повышены исходно. Сохраняющиеся повышенными уровни маркеров свидетельствует о наличии резидуальной опухоли.

\subsubsection{1. ЛЕЧЕНИЕ ДИСГЕРМИНОМЫ}

ІА стадия. При адекватном стадировании и подтверждении ІАстадии дисгерминомы яичников рекомендовано динамическое наблюдение (график наблюдения - см. пункт 3.2.1.3).

IB-IC стадии. При IB-IC стадии дисгерминомы, а также при клинической I стадии без адекватного хирургического стадирования или в случае отказа от динамического наблюдения рекомендуется проведение 3 курсов ХТ по схеме ВЕР либо 2 цикла адъювантной ХТ карбоплатином AUC7 в монорежиме.

IIA-IV стадии. Рекомендовано проведение 3 циклов XТ по схеме ВЕР. При уровне лДГ >2 ВГН или при наличии висцеральных метастазов рекомендуется проведение 4 циклов ВЕР. В связи с риском поздней токсичности блеомицина в виде пульмонита рекомендовано исключить блеомицин из схемы терапии при лечении пациенток старше 40 лет, а также в случае ранее перенесенных заболеваний легких; для этой категории рекомендуется проведение 4 циклов ЕР. 


\subsubsection{2. ЛЕЧЕНИЕ НЕЗРЕЛОЙ ТЕРАТОМЫ}

IA стадия, G1 степень дифференцировки. При адекватном стадировании и подтверждении IA G1 стадии незрелой тератомы яичников рекомендовано динамическое наблюдение (график наблюдения - см. пункт 3.2.1.3).

IA стадия, G2-G3 степени дифференцировки. Рекомендовано назначение 3 циклов ХТ по схеме ВЕР.

IC-IV стадии. Рекомендовано проведение XТ по схеме ВЕР. Количество циклов зависит от наличия резидуальной опухоли после хирургического лечения: при отсутствии резидуальной опухоли показаны 3 цикла ХТ по схеме ВЕР, при наличии остаточной опухоли рекомендовано проведение 4 циклов по схеме ВЕР. Наличие повышенных уровней опухолевых маркеров АФП или $\beta$-ХГЧ свидетельствует о наличии резидуальной опухоли.

\subsubsection{3. ЛЕЧЕНИЕ ОПУХОЛИ ЖЕЛТОЧНОГО МЕШКА,}

ХОРИОКАРЦИНОМЫ, ЭМБРИОНАЛЬНОГО РАКА, ПОЛИЭМБРИОМЫ, СМЕШАННОЙ ЗЛОКАЧЕСТВЕННОЙ ГЕРМИНОГЕННОЙ ОПУХОЛИ

Опухоль желточного мешка, хориокарцинома, эмбриональный рак, полиэмбриома, смешанная злокачественная герминогенная опухоль являются высокозлокачественными опухолями, в связи с чем назначение ХТ является обязательным при любой стадии заболевания. Больным с ранними стадиями и/или отсутствием резидуальной опухоли рекомендованы 3 цикла ХТ по схеме ВЕР, при наличии резидуальной опухоли рекомендуются 4 цикла по схеме BEP.

Таблица 3. Тактика лечения больных ГОЯ после хирургического стадирования

\begin{tabular}{|l|l|l|l|l|}
\hline \multirow{2}{*}{ Тип ГОя } & \multicolumn{4}{l|}{ Стадия заболевания } \\
\cline { 2 - 6 } & IA G1 & IA G2-3 & IB-IC & IIA-IV \\
\hline & & & & \\
\hline Дисгерминома & & & & \\
\hline Незрелая тератома & Наблюдение & & $\begin{array}{l}\text { карбоплатина } \\
\text { АUС7 }\end{array}$ & 3 цикла ВEP \\
\hline $\begin{array}{l}\text { Опухоль желточного мешка, } \\
\text { хориокарцинома, эмбриональный } \\
\text { рак, смешанная злокачественная } \\
\text { герминогенная опухоль }\end{array}$ & Наблюдение & 3 цикла ВЕР & & 3-4 цикла ВЕР1 \\
\hline
\end{tabular}

1 При отсутствии резидуальной опухоли рекомендованы 3 курса XT по схеме BEP, при наличии резидуальной опухоли рекомендованы 4 курса ХT по схеме ВEP. 


\subsubsection{3. Принципы динамического наблюдения после хирургического лечения при I стадии дисгерминомы и G1 незрелой тератомы}

Рекомендовано тщательное динамическое наблюдение согласно графику при условии выполненного хирургического стадирования.

График наблюдения при дисгерминоме (проводится в условиях поликлиники территориального онкологического диспансера или у районного онколога): физикальный и гинекологический осмотр, уровни $\beta$-ХГЧ, ЛДГ; УЗИ органов брюшной полости и малого таза - каждые 3 мес. в течение 1-го года; каждые 4 мес.- в течение 2-го и 3-го года; каждые 6 мес.- в течение 4-го года; далее - ежегодно (до 10 лет). Рентгенография органов грудной клетки выполняется каждые 6 мес. в течение первых 2 лет, далее - ежегодно (до 5 лет).

График наблюдения при незрелой тератоме: физикальный и гинекологический осмотр, уровни АФП, $\beta$-ХГЧ - каждые 1-2 мес.; УЗИ органов брюшной полости и малого таза - каждые 2 мес. в течение 1-го года; каждые 3 мес.- в течение 2-го и 3-го года; каждые 6 мес.в течение 4-го года; далее - ежегодно (до 10 лет). Рентгенография органов грудной клетки выполняется каждые 6 мес. в течение первых 2 лет, далее - ежегодно (до 5 лет).

\subsection{2. Принципы проведения химиотерапии}

Начало ХТ рекомендовано в ближайшие сроки (10-14 дней) после проведения хирургического этапа лечения и стадирования. В табл. 4 представлены режимы адъювантной или ХТ 1-й линии ЗГОЯ.

При противопоказаниях к назначению блеомицина возможно проведение XT по программе ЕР или PEI в количестве 3-4 циклов в зависимости от наличия остаточной опухоли (при дисгерминоме в случае отсутствия резидуальной опухоли возможной альтернативой являются 3 цикла $E P$, при наличии опухоли - 4 цикла ЕР). У пациенток с распространенной недисгерминомой комбинация РЕІ (этопозид, цисплатин, ифосфамид) сопоставима по эффективности с режимом ВЕР, но является более миелотоксичной. При отсутствии резидуальной опухоли показаны 3 цикла XТ по схеме PEI, при ее наличии - 4 цикла по схеме PEI.

Вследствие развития поздней токсичности блеомицина в виде пульмонитов рекомендовано исключить блеомицин из схемы терапии у пациенток старше 40 лет, а также в случае хронических заболеваний легких.

Все циклы ХТ проводятся каждые 3 недели (считая от начала предыдущего курса XT). Возможна задержка очередного курса лечения при наличии инфекции, нейтропении с АЧН < 0,5×109/л менее или тромбоцитопении с числом тромбоцитов <50,0×109/л в первый день планируемого курса (см. табл. 5). При развитии фебрильной нейтропении, нейтропении IV cт. длительностью свыше 7 дней или нейтропении, осложненной инфекцией, обосновано профилактическое назначение Г-КСФ при проведении всех последующих циклов ХТ.

У пациентов в тяжелом общем состоянии, обусловленном распространенностью опухолевого процесса, первый цикл ХТ может быть проведен с редукцией доз препаратов

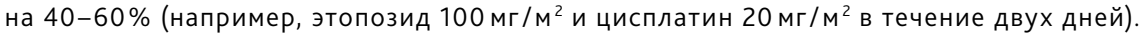
Режимы ХТ указаны в табл. 4. Необходимо перед каждым циклом ХТ определять опухолевые маркеры, по окончании ХТ - повторить КТ исходных зон поражения. 
У пациентов с недисгерминомой увеличение в размерах или появление новых очагов

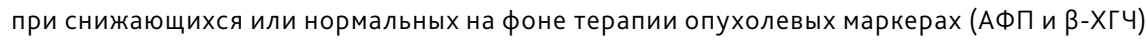
вероятнее всего является синдромом растущей зрелой тератомы, а не признаком прогрессирования. В данных ситуациях необходимо завершение запланированного объема ХT с последующим хирургическим удалением опухоли.

\subsection{3. Лечение остаточной опухоли после химиотерапии}

\subsubsection{1. Дисгерминома}

При наличии остаточной опухоли (по брюшине, в забрюшинных лимфоузлах, легких, лимфоузлах средостения и др.) после завершения ХТ и нормализации опухолевых маркеров дальнейшее лечение не показано, проводится динамическое наблюдение. При размере остаточной опухоли > 3 см возможно выполнение ПЭТ/КТ (не ранее чем через 8 нед. после завершения ХТ). При патологическом накоплении РФП в остаточной опухоли рекомендовано ее удаление. При отказе пациентки или технической невозможности хирургического лечения показано динамическое наблюдение.

\subsubsection{2. Недисгерминома (опухоль желточного мешка, эмбриональный рак, хориокарцинома, полиэмбриома, незрелая тератома, смешанная герминогенная опухоль)}

При наличии остаточной опухоли (по брюшине, в забрюшинных лимфоузлах, легких, лимфоузлах средостения и др.) <1 см после завершения ХТ и нормализации опухолевых маркеров дальнейшее лечение не показано, проводится динамическое наблюдение. При размере остаточной опухоли $\geq 1$ см показано ее удаление. При синдроме растущей зрелой тератомы рекомендовано хирургическое удаление опухоли, XT не показана.

При обнаружении в остаточной опухоли элементов злокачественной герминогенной опухоли показана XT второй линии (режимы TIP или VelP): 2 цикла - в случае радикального объема операции, 4 цикла - при наличии остаточной опухоли.

\subsection{4. Лечение рецидивов ЗГОЯ}

Перед началом ХТ рецидива необходимо исключить синдром растущей зрелой тератомы - появление или увеличение в размерах опухолевыхузлов на фоне снижающихся/нормальных опухолевых маркеров. В данной ситуации показано хирургическое лечение в объеме радикального удаления всех опухолевых узлов. Как правило, при наличии остаточной опухоли происходит рост узлов, что требует повторных операций. При подтверждении зрелой тератомы при гистологическом исследовании удаленного препарата проведение системного лекарственного лечения не требуется.

Стандартной ХT рецидива являются комбинации на основе ифосфамида и цисплатина (табл. 4). Оптимальным является режим TIP, альтернативой ему может служить режим VeIP. Обычно проводятся 4 цикла. Не показано преимуществ того или иного режима в качестве второй линии терапии. Применение высокодозной ХТ с поддержкой костномозгового 
кроветворения по данным небольших наблюдений позволяет достичь обнадеживающих результатов и может применяться во II-III линиях в центрах, имеющих опыт ее проведения. После завершения XT показано хирургическое вмешательство с целью максимального удаления остаточной опухоли.

В случае роста маркеров, несмотря на проводимую терапию, исчерпанности возможностей XT, локализации опухоли в одной анатомической области возможна попытка ее удаления. Этот подход дает шанс излечить около $25 \%$ пациентов, особенно с поздними рецидивами, умеренно повышенным уровнем АФП и забрюшинной локализацией резидуальной опухоли. При бурном прогрессировании с ростом $\beta$-ХГЧ оперативное лечение не показано. У пациенток с высоким уровнем MSI в качестве третьей и последующих линий терапии возможно применение иммунотерапии (пембролизумаб, ниволумаб или комбинации ниволумаба с ипилимумабом).

В лечении поздних рецидивов, возникших по прошествии двух и более лет после окончании предшествующей ХT, основным методом лечения является хирургический. Особенностью поздних рецидивов является низкая чувствительность к XТ, что позволяет рекомендовать в случае потенциально резектабельных опухолей на первом этапе выполнять хирургическое лечение даже в случае повышенных маркеров. При невозможности радикального удаления опухоли и повышенных маркерах необходимо начинать ХТ второй линии с последующим выполнением операции.

\section{3. Редкие опухоли яичников}

\subsection{1. Нейроэндокринные опухоли яичников (мелкоклеточный рак яичников и карциноиды яичников)}

Нейроэндокринные опухоли отличаются экспрессией одного или нескольких нейроэндокринных маркеров, таких как хромогранин, синаптофизин, нейронспецифическая енолаза. Мелкоклеточный рак яичников характеризуется агрессивным течением, морфологически схож с мелкоклеточным раком легкого. Стандартным хирургическим вмешательством является оптимальная циторедуктивная операция (абдоминальная гистерэктомия с билатеральной сальпингоовариэктомией и оментэктомией) с последующей адъювантной XT, аналогичной таковой при мелкоклеточном раке легкого. Гиперкальциемический тип мелкоклеточного рака яичников часто сочетается с гиперкальциемией, гипонатриемией и задержкой жидкости. В качестве симптоматического лечения в таких ситуациях могут использоваться:

- регидратация 0,9\% раствором натрия хлорида;

- терапия бисфосфонатами;

- солевой диурез: 0,9\% раствор натрия х лорида +диуретики (фуросемид).

Карциноиды яичника - это высокодифференцированные нейроэндокринные опухоли с менее агрессивным течением, которые часто ошибочно принимают за метастазы в яичниках злокачественных опухолей ЖКТ (в первую очередь необходимо исключить первичную опухоль ЖКТ). Молодым женщинам при одностороннем поражении яичника 
может быть предложена органосохраняющая операция, в остальных случаях выполняется оптимальная циторедуктивная операция (абдоминальная гистерэктомия с билатеральной сальпингоовариэктомией и оментэктомией). При наличии карциноидного синдрома назначаются аналоги соматостатина. Принципы лечения карциноидов яичника схожи с лечением высокодифференцированных нейроэндокринных опухолей ЖКТ и изложены в соответствующем разделе.

\subsection{2. Карциносаркомы яичников}

Это редкий вариант прогностически неблагоприятных злокачественных новообразований яичников, на долю которых приходится не более 2-4\% всех злокачественных опухолей яичников. Важным этапом в диагностике карциносаркомы яичников, подобно карциносаркоме матки, является определение соотношения эпителиального и мезенхимального компонентов в опухолевой ткани. В случае преобладания эпителиального компонента лекарственное лечение должно быть аналогичным таковому при раке яичников (используются препараты платины и таксаны), а при преобладании мезенхимального компонента - схожим слечением сарком мягких тканей (см. раздел по лечению сарком мягких тканей). После циторедуктивной операции (абдоминальная гистерэктомия с билатеральной сальпингоовариэктомией и оментэктомией, хирургическим стадированием) в оптимальном объеме (по возможности) всем пациенткам независимо от стадии показано проведение 4-6 циклов адъювантной XT (табл. 4), выбор которой определяется преобладающим компонентом (эпителиальный или мезенхимальный). При возникновении рецидива также важна морфологическая верификация с планированием XТ в зависимости от преобладающего компонента.

Таблица 4. Рекомендуемые режимы лекарственной терапии злокачественных неэпителиальных опухолей яичников

\begin{tabular}{|c|c|}
\hline \multicolumn{2}{|r|}{ 1. Режимы адъювантной ХТ при опухолях стромы полового тяжа и стероидноклеточных опухолях } \\
\hline BEP & 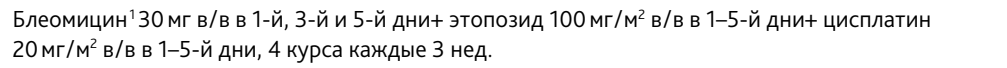 \\
\hline CPtx & Карбоплатин AUC-6 в/в в 1-й день+ паклитаксел 175 мг/м² в/в в 1-й день, каждые 3 нед. 6 курсов \\
\hline EP & Этопозид 100 мг/м² в/в в 1-5-й дни+ цисплатин 20 мг/м² в/в в 1-5-й дни, каждые 3 нед. 4 курса \\
\hline \multicolumn{2}{|r|}{$\begin{array}{l}\text { 2. Режимы XТ при распространенных стадиях и рецидивах опухолей стромы полового тяжа и стероидно- } \\
\text { клеточных опухолях (у больных, получавших ранее режим ВЕР) }\end{array}$} \\
\hline CPtx & $\begin{array}{l}\text { Карбоплатин AUC-6 в/в в 1-й день+ паклитаксел } 175 \text { мг/м² в/в в 1-й день, 4-6 циклов каждые } \\
3 \text { нед. }\end{array}$ \\
\hline TIP & 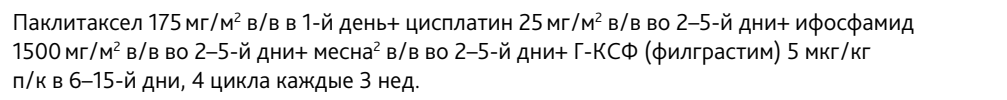 \\
\hline $\mathrm{CDE}$ & 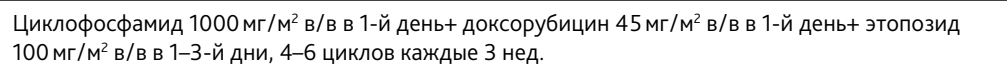 \\
\hline CAV & 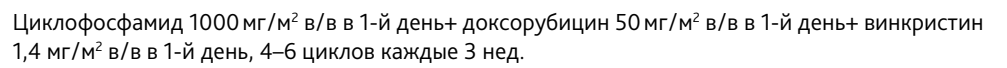 \\
\hline
\end{tabular}


3. Режимы лекарственной терапии II-III линий при распространенных стадиях и рецидивах опухолей стромы полового тяжа и стероидноклеточных опухолях

Ифосфамид 1,6 мг/м² в/в в 1-3-й дни 1-часовая инфузия+ месна² в/в с 1-3-й дни+ паклитаксел 175 мг/м² в/в в 3-й день каждые 3 нед.

Доцетаксел 75 мг/м² в/в каждые 3 нед.

Бевацизума6 7,5-15 мг/кг в/в каждые 3 нед.

Тамоксифен 20 мг внутрь 2 раза в сутки ежедневно

Ингибиторы ароматазы:

- летрозол 2,5 мг/сут. внутрь ежедневно

- анастрозол 1 мг/сут. внутрь ежедневно

- эксеместан 25 мг/сут. внутрь ежедневно

Аналоги ГРГ:

- лейпрорелин 3,75 мг в/м 1 раз в 28 дней

- гозерелин 3,6 мг п/к 1 раз в 28 дней или 10,8 мг п/к 1 раз в 84 дня

- бусерелин 3,75 мг в/м 1 раз в 28 дней

4. Рекомендуемые режимы XТ при герминогенных опухолях яичников

\section{І линия}

ВЕР 20 мг/м² в/в в 1-5-й дни, 3-4 цикла каждые 3 нед.

EP Этопозид 100 мг/м² в/в в 1-5-й дни+ цисплатин 20 мг/м² в/в в 1-5-й дни, 3-4 цикла каждые 3 нед.

PEI Ифосфамид 1200 мг/м² в/в в 1-5-й дни+ месна ${ }^{2}$ в/в со 2-5 дни+ этопозид

$75 \mathrm{мг} / \mathrm{M}^{2}$ в/в в 1-5-й дни+ цисплатин 20 мг/м² в/в в 1-5-й дни+Г-КСФ (филграстим) 5 мкг/кг п/к в 6-12-й дни, 3-4 цикла каждые 3 нед.

\section{II линия}

\begin{tabular}{|c|c|}
\hline TIP & 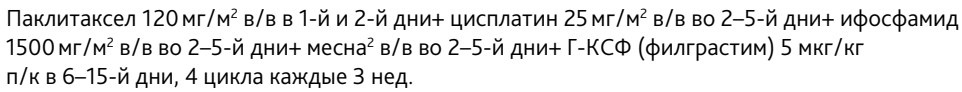 \\
\hline VelP & $\begin{array}{l}\text { Винбластин 0,11 мг/кг в/в в 1-й и 2-й дни+ ифосфамид } 1200 \text { мг/м² в 1-5-й дни+ мес- } \\
\text { на² в 1-5-й дни+ цисплатин } 20 \text { мг/м² в/в в 1-5-й дни+ Г-КСФ (филграстим) } 5 \text { мкг/кг } \\
\text { п/к в 6-15-й дни, } 4 \text { цикла каждые } 3 \text { нед. }\end{array}$ \\
\hline \multicolumn{2}{|c|}{ III линия } \\
\hline TGO & 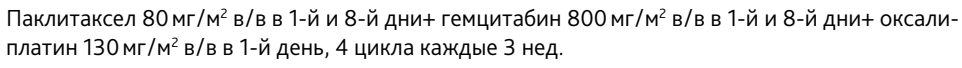 \\
\hline GemOx & $\begin{array}{l}\text { Гемцитабин } 1000 \text { мг/м² в/в в 1-й и 8-й дни+ оксалиплатин } 130 \text { мг/м² в/в в 1-й день, } 4 \text { цикла } \\
\text { каждые } 3 \text { нед. }\end{array}$ \\
\hline
\end{tabular}

1 Блеомицетина гидрохлорид (изомер А5) и блеомицина сульфат (смесь изомеров А2 и В2) не являются идентичными лекарственными средствами. При использовании блеомицина сульфата в режиме ВЕР

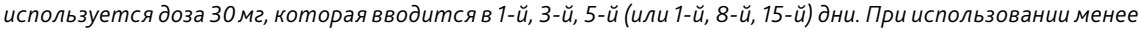
изученного блеомицетина гидрохлорида рекомендуется снижение курсовой дозы на 30-40\%, например, по 30 мг в 1-й и 5-й дни. Введение цисплатина осуществляется на фоне в/в гидратации физиологическим раствором хлорида натрия (суммарный суточный объем 2,5 л), необходимой для поддержания диуреза > 100 мл/час в процессе введения цисплатина и в последующие 3 часа; 
2 Месна применяется в суточной дозе, составляющей $100 \%$ от дозы ифосфамида и разделенной на 3 введения в течение дня: непосредственно перед введением ифосфамида и далее через 4 и 8 часов после начала его инфузии. Возможна замена второго и третьего (через 4 и 8 часов) в/в введения месны на пероральный. Для этого она применяется в большей разовой дозе (40\% от дозы ифосфамида) в виде раствора в Кока-коле или соке в соотношении от 1:1 до 1:10.

Таблица 5. Схема редукции доз препаратов в режимах ВЕР/ЕР при гематологической токсичности (на основе показателей общеклинического анализа крови на 21-й день от начала предыдущего курса)

\begin{tabular}{|c|c|c|c|c|c|c|c|c|}
\hline \multirow{2}{*}{$\begin{array}{l}\text { Количество } \\
\text { тромбоцитов } \\
(\times 109 / л)\end{array}$} & \multicolumn{2}{|l|}{$\geq 100,0$} & \multicolumn{2}{|l|}{$75-99$} & \multicolumn{2}{|l|}{$50-74$} & \multicolumn{2}{|l|}{$<50$} \\
\hline & $\begin{array}{l}\text { Этопо- } \\
\text { зид }\end{array}$ & $\begin{array}{l}\text { Цис- } \\
\text { платин }\end{array}$ & $\begin{array}{l}\text { Этопо- } \\
\text { зид }\end{array}$ & $\begin{array}{l}\text { Цис- } \\
\text { платин }\end{array}$ & $\begin{array}{l}\text { Этопо- } \\
\text { зид }\end{array}$ & $\begin{array}{l}\text { Цис- } \\
\text { платин }\end{array}$ & $\begin{array}{l}\text { Этопо- } \\
\text { зид }\end{array}$ & $\begin{array}{l}\text { Цис- } \\
\text { платин }\end{array}$ \\
\hline$\geq 1,0$ & $100 \%$ & $100 \%$ & $75 \%$ & $100 \%$ & $50 \%$ & $100 \%$ & \multicolumn{2}{|c|}{ Отсрочка на 4 дня } \\
\hline $0,5-0,99$ & $100 \%$ & $100 \%$ & $50 \%$ & $100 \%$ & \multicolumn{2}{|c|}{ Отсрочка на 4 дня } & \multicolumn{2}{|c|}{ Отсрочка на 4 дня } \\
\hline$<0,5$ & \multicolumn{2}{|c|}{ Отсрочка на 4 дня } & \multicolumn{2}{|c|}{ Отсрочка на 4 дня } & \multicolumn{2}{|c|}{ Отсрочка на 4 дня } & \multicolumn{2}{|c|}{ Отсрочка на 4 дня } \\
\hline
\end{tabular}

\section{4. НАБЛЮДЕНИЕ}

\section{1. Злокачественные герминогенные опухоли}

С учетом высокой курабельности герминогенных опухолей и длительной ожидаемой продолжительности жизни необходима профилактика и ранняя диагностика поздних осложнений ХТ (сердечно-сосудистые заболевания, метаболический синдром, гипогонадизм, инфертильность, легочная токсичность и др.) с привлечением соответствующих профильных специалистов. Для больных герминогенными опухолям яичников после проведенной ХТ рекомендуется:

- физикальный осмотр, определение опухолевых маркеров, УзИ органов брюшной полости, забрюшинного пространства и пахово-подвздошных областей - каждые 2-3 мес. в первый год, каждые 3 мес.- во второй год, каждые 4 мес.- в третий и четвертый годы, раз в полгода - пятый год и далее - ежегодно;

- рентгенография органов грудной клетки выполняется каждые 6 мес. в первые два года, далее - ежегодно в течение 5 лет.

\section{2. Опухоли стромы и полового тяжа}

ОСПТ низкого риска, ранние стадии: физикальный осмотр, определение опухолевых маркеров (ингибин, антимюллеров гормон, эстрадиол, тестостерон - в зависимости от гистологической формы опухоли), УзИ органов брюшной полости, забрюшинного пространства и пахово-подвздошных областей - каждые 6-12 мес. в течение 10 и более лет. 
ОСПТ, ранние стадии высокого риска и распространенные стадии: физикальный осмотр, опухолевые маркеры (ингибин, антимюллеров гормон, эстрадиол, тестостерон - в зависимости от гистологической формы опухоли), УЗИ органов брюшной полости, забрюшинного пространства и пахово-подвздошных областей - каждые 4-6 мес. в течение 10 и более лет.

Рентгенография органов грудной клетки - 1 раз в год; КГ органов брюшной полости и/или грудной клетки - по показаниям.

\section{3. Редкие опухоли яичников}

Физикальный осмотр, опухолевые маркеры (СА125 и др.-в зависимости от гистологической формы опухоли), УзИ органов брюшной полости, забрюшинного пространства и пахово-подвздошных областей - каждые 3 мес. в течение первых 3 лет, в последующие годы 1 раз в 4-6 мес. КТ/МРТ брюшной полости с контрастированием - по показаниям. Рентгенография органов грудной клетки 1 раз в 6 мес. в течение первых 3 лет, далее - 1 раз в год. 